\title{
Distribution of incompressible flow within interdigitated channels and porous electrodes
}

\author{
Robert J. Kee ${ }^{\mathrm{a}, *}$, Huayang Zhu ${ }^{\mathrm{a}}$ \\ ${ }^{a}$ Mechanical Engineering, Colorado School of Mines, Golden, CO 80401, \\ USA
}

\begin{abstract}
This paper develops a general model with which to evaluate flow uniformity and pressure drop within interdigitated-channel structures, especially in the context of redox flow batteries. The governing equations are cast in dimensionless variables, leading to a set of characteristic dimensionless parameter groups. The systems of governing equations are solved computationally, with the results presented graphically. Because the results are general, the underlying model itself is not needed to apply the quantitative design guidelines. However, the paper presents and discusses all the information required to recreate the model as needed.

Keywords:

Interdigitated channels, Flow battery, Fuel cell, Porous media, Design guidelines
\end{abstract}

\footnotetext{
*Corresponding author. Tel: +1-303-273-3379.

Email address: rjkee@mines.edu (Robert J. Kee)
} 


\section{Introduction}

Redox flow batteries, polymer-electrolyte fuel cells (PEMFC), and potentially other technologies can be designed with interdigitated channels that supply flow through underlying porous electrode structures. Device performance often depends on tradeoffs between flow uniformity over large areas and overall pressure losses.

The objective of the present paper is to develop quantitative guidelines that assist the design of interdigitated-channel configurations and operating conditions. The approach is based upon a combination of models that are formulated in terms of characteristic dimensionless groups. The results are presented graphically, revealing quantitative tradeoffs between flow uniformity and pressure drop. The model results are reduced to a general set of design guidelines that do not require running the model for particular geometric configurations or operating conditions.

Figure 1 illustrates some of the salient features of an interdigitated redox flow battery layout. Small rectangular flow channels are situated within the bipolar plates. The "feed" channels are completely closed at the exit end of the structure, and "exhaust" channels are completely closed at the entrance end of the structure. Thus, all the fluid (electrolyte) that enters the feed channels must flow through the porous electrodes and leave via the exhaust channels. Electrochemical reactions proceed within the porous electrodes, with ion mobility across the membrane that separates the anode and cathode.

A variety of channel layouts, including interdigitated channels, are used in redox flow batteries [1-5] and fuel cells [6-16]. Much of the modeling literature is based on various approaches for two- and three-dimensional compu- 
tational fluid dynamics. Some investigations specifically consider under-rib convection, especially for PEMFC serpentine designs [17-19]. Most models also incorporate electrochemistry as well as ion mobility across the membrane that separates anode and cathode. In essentially all cases, the physical problem is solved directly, without an attempt to generalize in dimensionless terms.

The present paper is devoted entirely to flow distribution; it does not consider chemistry or electrochemistry within the porous structures. Flow batteries are often designed with relatively low single-pass conversion within the porous electrode structures. Thus, it is reasonable to neglect the effects of electrode chemistry and membrane transport when considering the flow distribution. The important contribution of the present approach is to develop generalized, but quantitative, guidelines for the flow distribution.

Although the results of the present analysis are general, there are also limitations based upon physical assumptions. The model is certainly compromised if the mass transport across the ion-transport membrane (cf., Fig. 1), is comparable to the mass transport between channels. The model assumes fully developed flow within the channels (i.e., entry-length effects are neglected). Laminar flow is typical in flow-battery channels, but the model is not restricted to laminar flow. For gas-phase flows (e.g., as in PEMFC), the incompressibility assumption can be compromised. Although including an equation of state in the model itself is easily done, doing so limits the generality of the dimensionless results. Finally, molecular diffusion is neglected. In applications such as PEMFC fuel cells with gaseous fuels, axial diffusive transport within the channels and molecular diffusion within the 
gas-diffusion structures can play important roles.

The model focuses specifically on two interdigitated channels and the underlying porous structure. Thus, there is an implicit assumption that each channel pair within a full battery layout behaves the same as all others. In other words, the manifold or header structures are designed to feed all the interdigitated channels equally.

The theoretical and computational approach developed here has its foundation in earlier models for flow distribution in parallel-flow header-channel configurations [20]. The new elements of the present paper are related to the generalization of flow through porous electrode structures that connect the interdigitated channels. The present model and the dimensionless results provide easy-to-use design guidance. Nevertheless, it is also clear that complete design process benefits greatly from much more detailed models that incorporate chemical, electrochemical, and thermal behaviors.

\section{Channel flow}

Assuming steady-state, single-component, isothermal, incompressible, plug flow, the mass-continuity equations within the channels can be written as

$$
\rho \frac{\mathrm{d} u_{\mathrm{f}}}{\mathrm{d} z}=-\frac{K\left(p_{\mathrm{f}}-p_{\mathrm{e}}\right)}{H_{\mathrm{c}}},
$$

$$
\rho \frac{\mathrm{d} u_{\mathrm{e}}}{\mathrm{d} z}=+\frac{K\left(p_{\mathrm{f}}-p_{\mathrm{e}}\right)}{H_{\mathrm{c}}},
$$

where $u_{\mathrm{f}}$ and $u_{\mathrm{e}}$ are mean velocities in the feed and exhaust channels, respectively. The rectangular channels are assumed to be $W_{\mathrm{c}}$ wide, $H_{\mathrm{c}}$ high, and $L_{\mathrm{c}}$ long. The independent variable $z$ is the axial position within the channels. The mass flux between adjacent channels (i.e., through the underlying 
73

porous media) can be represented as

$$
m^{\prime \prime}=K\left(p_{\mathrm{f}}-p_{\mathrm{e}}\right),
$$

74

where $p_{\mathrm{f}}$ and $p_{\mathrm{e}}$ are the local pressures within the feed and exhaust channels, respectively. The "flux" is referenced to the surface area on the floor of the channels. The parameter $K$ is a function of the porous-media properties and physical dimensions, but is assumed to be a constant for any particular system.

The axial momentum equations within the channels can be written as

$$
\frac{\mathrm{d}\left(\rho u_{\mathrm{f}} u_{\mathrm{f}}\right)}{\mathrm{d} z}=-\frac{\mathrm{d} p_{\mathrm{f}}}{\mathrm{d} z}-\tau_{\mathrm{w}} \frac{P_{\mathrm{c}}}{A_{\mathrm{c}}}
$$

$$
\frac{\mathrm{d}\left(\rho u_{\mathrm{e}} u_{\mathrm{e}}\right)}{\mathrm{d} z}=-\frac{\mathrm{d} p_{\mathrm{e}}}{\mathrm{d} z}-\tau_{\mathrm{w}} \frac{P_{\mathrm{c}}}{A_{\mathrm{c}}}
$$

where $\tau_{\mathrm{w}}$ is the local shear stress at the channel walls, $P_{\mathrm{c}}=2\left(W_{\mathrm{c}}+H_{\mathrm{c}}\right)$ is the channel's wetted perimeter, and $A_{\mathrm{c}}=W_{\mathrm{c}} H_{\mathrm{c}}$ is the channel's cross-sectional area. Because the mass flux between the channels is assumed to be normal to the axial direction, a mass-flux term does not directly appear in the axial momentum equations. However, the mass flux is indirectly involved via the velocity coupling with the continuity equations.

The wall shear stress can be represent in terms of a friction factor as

$$
f=\frac{\tau_{\mathrm{w}}}{\rho u^{2} / 2}
$$

For fully developed laminar flow in a rectangular channel, the friction factor can be correlated in terms of a Reynolds number as

$$
\operatorname{Re} f=13.74+10.38 \exp \left(-\frac{3.4}{\alpha}\right)
$$


where $\alpha=H_{\mathrm{c}} / W_{\mathrm{c}}(0 \leq \alpha \leq 1)$ is the channel aspect ratio [20,21]. The Reynolds number $\operatorname{Re}=\rho u D_{\mathrm{h}} / \mu$ is evaluated based upon the mean velocity and the channel hydraulic diameter $D_{\mathrm{h}}=4 A_{\mathrm{c}} / P_{\mathrm{c}}$. The fluid's dynamic viscosity is represented as $\mu$. Although Eq. 7 is a laminar friction-factor correlation, the dimensionless analysis does not depend upon the particular correlation. Any other representation of the $\operatorname{Re} f$ (e.g., representing turbulent flow) is fully accommodated in the analysis.

The two momentum equations can be summarized as

$$
2 \rho u_{\mathrm{f}} \frac{\mathrm{d} u_{\mathrm{f}}}{\mathrm{d} z}=-\frac{\mathrm{d} p}{\mathrm{~d} z}-\left(\frac{P_{\mathrm{c}}}{2 A_{\mathrm{c}} D_{\mathrm{h}}} \mu \operatorname{Re} f\right) u_{\mathrm{f}} .
$$

$$
2 \rho u_{\mathrm{e}} \frac{\mathrm{d} u_{\mathrm{e}}}{\mathrm{d} z}=-\frac{\mathrm{d} p}{\mathrm{~d} z}-\left(\frac{P_{\mathrm{c}}}{2 A_{\mathrm{c}} D_{\mathrm{h}}} \mu \operatorname{Re} f\right) u_{\mathrm{e}}
$$

The interdigitated-channel problem can be generalized by writing the equations in dimensionless variables. A set of dimensionless variables can be defined as

$$
\hat{z}=\frac{z}{L_{\mathrm{c}}}, \quad \hat{u}_{\mathrm{f}}=\frac{u_{\mathrm{f}}}{U_{\mathrm{in}}}, \quad \hat{u}_{\mathrm{e}}=\frac{u_{\mathrm{e}}}{U_{\mathrm{in}}},
$$

$$
\hat{p}_{\mathrm{f}}=\frac{p_{\mathrm{f}}}{\rho U_{\mathrm{in}}^{2}}, \quad \hat{p}_{\mathrm{e}}=\frac{p_{\mathrm{e}}}{\rho U_{\mathrm{in}}^{2}} .
$$

The inlet velocity for the feed channel is specified as $U_{\text {in }}$.

Following transformation to the dimensionless form, the system of equations can be summarized as

$$
\frac{\mathrm{d} \hat{u}_{\mathrm{f}}}{d \hat{z}}=-\Omega\left(\hat{p}_{\mathrm{f}}-\hat{p}_{\mathrm{e}}\right)
$$

$$
\frac{\mathrm{d} u_{\mathrm{e}}}{d \hat{z}}=+\Omega\left(\hat{p}_{\mathrm{f}}-\hat{p}_{\mathrm{e}}\right) .
$$

$$
2 \hat{u}_{\mathrm{f}} \frac{\mathrm{d} \hat{u}_{\mathrm{f}}}{\mathrm{d} \hat{z}}=-\frac{\mathrm{d} \hat{p}}{\mathrm{~d} \hat{z}}-\Gamma \hat{u}_{\mathrm{f}},
$$


108

111

$$
2 \hat{u}_{\mathrm{e}} \frac{\mathrm{d} \hat{u}_{\mathrm{e}}}{\mathrm{d} \hat{z}}=-\frac{\mathrm{d} \hat{p}}{\mathrm{~d} \hat{z}}-\Gamma \hat{u}_{\mathrm{e}}
$$

The dimensionless equations contain two dimensionless groups of physical and fluid parameters:

$$
\Omega \equiv \frac{L_{\mathrm{c}}}{H_{\mathrm{c}}} K U_{\mathrm{in}},
$$

$$
\Gamma \equiv \frac{P_{\mathrm{c}}}{2 A_{\mathrm{c}} D_{\mathrm{h}}} \frac{L_{\mathrm{c}}}{U_{\text {in }}} \frac{\mu}{\rho} \operatorname{Re} f .
$$

Each of the governing equations is a first-order ordinary differential equation. Together, the system of equations is a fourth-order ordinary-differentialequation boundary-value problem. As such, four boundary conditions are needed for solution. At the inlet end (i.e., $\hat{z}=0$ ), the dimensionless mean velocities are written as

$$
\hat{u}_{\mathrm{f}}(0)=1, \quad \hat{u}_{\mathrm{e}}(0)=0 .
$$

Note that the exhaust channel is blocked at the inlet end. At the exit end (i.e., $\hat{z}=1$ ),

$$
\hat{u}_{\mathrm{f}}(1)=0, \quad \hat{p}_{\mathrm{e}}(1)=0 .
$$

The pressure at the end of the exhaust channel is specified as a reference pressure of zero. The feed channel is blocked at the exit end (i.e., $\hat{u}_{\mathrm{f}}(1)=0$ ). Note that three boundary conditions are specified for velocity, but only one for pressure. In other words, the pressure at the inlet of the feed channel is not specified directly, but is determined from the solution. Such an implicit boundary condition affects the computational solution algorithm.

The dimensionless parameters include physical dimensions, fluid properties (viscosity and mass density), operating parameters (velocity), a friction- 
141

142

143

factor correlation $(\operatorname{Re} f)$, and a porous-media parameter $K$. Typical operating conditions, channel dimensions, and electrolyte fluid properties are available in the literature (e.g., Darling and Perry [4]). As discussed subsequently, the $K$ parameter depends on the porous-media permeability. Permeability, which depends on the electrode material itself and compression load, is available from manufacturer's specifications (e.g., SIGRACELL ${ }^{\circledR}$ battery felt, SGL Group, Wiesbaden, Germany).

\section{Computational solution}

As illustrated by the stencil shown in Fig. 2, the differential equations are discretized using a finite-volume mesh network. Both the boundary conditions and the flow directions play central roles in the finite-difference discretization. The continuity equations are differenced in opposite directions, thus enabling the propagation of the velocity boundary conditions into the channel interiors. That is,

$$
\left(\frac{\hat{u}_{\mathrm{f}, j}-\hat{u}_{\mathrm{f}, j-1}}{\Delta \hat{z}}\right)+\Omega\left(\hat{p}_{\mathrm{f}, j}-\hat{p}_{\mathrm{e}, j}\right)=0, \quad(2 \leq j \leq J)
$$

$$
\left(\frac{\hat{u}_{\mathrm{e}, j+1}-\hat{u}_{\mathrm{e}, j}}{\Delta \hat{z}}\right)-\Omega\left(\hat{p}_{\mathrm{f}, j}-\hat{p}_{\mathrm{e}, j}\right)=0, \quad(1 \leq j \leq J-1),
$$

$$
2 \hat{u}_{\mathrm{f}, j}\left(\frac{\hat{u}_{\mathrm{f}, j}-\hat{u}_{\mathrm{f}, j-1}}{\Delta \hat{z}}\right)+\left(\frac{\hat{p}_{\mathrm{f}, j}-\hat{p}_{\mathrm{f}, j-1}}{\Delta \hat{z}}\right)+\Gamma \hat{u}_{\mathrm{f}, j}=0,
$$

$$
(2 \leq j \leq J)
$$

$$
2 \hat{u}_{\mathrm{e}, j}\left(\frac{\hat{u}_{\mathrm{e}, j}-\hat{u}_{\mathrm{e}, j-1}}{\Delta \hat{z}}\right)+\left(\frac{\hat{p}_{\mathrm{e}, j+1}-\hat{p}_{\mathrm{e}, j}}{\Delta \hat{z}}\right)+\Gamma \hat{u}_{\mathrm{e}, j}=0,
$$

$$
(1 \leq j \leq J-1) .
$$


In the momentum equations, note that the convective derivatives are both "upwinded" [21]. The formulation assumes that there is no flow reversal. That is, both $\hat{u}_{\mathrm{f}}$ and $\hat{u}_{\mathrm{e}}$ are always positive (i.e., proceeding from the feed end toward the exit end). Although in principle there could be local flow reversals, any flow reversal would indicate a very poor design. The pressure derivatives are differenced in the opposite direction from the velocity derivatives. The sense of the exhaust-channel pressure derivative propagates information from the specified exhaust pressure into the interior. Note, however, that the entrance pressure is not directly specified. Note also that the exhaust-channel convective derivative involves $\hat{u}_{\mathrm{e}, j-1}$, which is not defined at $j=1$. However, because $\hat{u}_{\mathrm{e}, 1}=0$, the entire term vanishes at the boundary.

Three of the boundary conditions are straightforward to implement. That is,

$$
\hat{u}_{\mathrm{f}, J}=0, \quad \hat{u}_{\mathrm{e}, 1}=0, \quad \hat{p}_{\mathrm{e}, J}=0 .
$$

The velocity boundary conditions are nominally associated with the continuity equations and the pressure boundary conditions are nominally associated with the momentum equations. This is a coupled boundary-value problem, where there is no need to directly associate the boundary conditions with governing equations. If the feed-channel inlet velocity is specified, then an implicit boundary condition is needed for the pressure. That is, the boundary condition that is nominally associated with pressure (i.e., the momentum equation) at the inlet is

$$
\hat{u}_{\mathrm{f}, 1}=1.0 \text {. }
$$

Following discretization, the computational problem is one of solving a system of nonlinear algebraic equations. The solution is represented as the 
values for velocity and pressure at each of the finite-volume mesh cells. A range of numerical-solution approaches can be used. In essentially all cases, the discrete equations are written in residual form. That is, for example, consider the feed-channel continuity equation

$$
\left(\frac{\hat{u}_{\mathrm{f}, j}-\hat{u}_{\mathrm{f}, j-1}}{\Delta \hat{z}}\right)+\Omega\left(\hat{p}_{\mathrm{f}, j}-\hat{p}_{\mathrm{e}, j}\right)=R_{\mathrm{f}, j}
$$

Each equation has such a residual form. The solution algorithm iterates on the values of the velocities and pressures until some norm of the residual vector is reduced to a sufficiently small value. At this point, the solution is found. The present study uses the MatlaB function "fsolve" to accomplish the solution using a Levenberg-Marquardt algorithm.

Given values of $\Omega$ and $\Gamma$, the solution to the dimensionless channel problem produces profiles of $\hat{u}_{\mathrm{f}}(\hat{z}), \hat{u}_{\mathrm{e}}(\hat{z}), \hat{p}_{\mathrm{f}}(\hat{z})$, and $\hat{p}_{\mathrm{f}}(\hat{z})$. From these solutions, two important summary metrics can be easily extracted. The first is the net pressure drop between the feed-channel inlet and the exhaust-channel outlet,

$$
\Delta \hat{p}=\hat{p}_{\mathrm{f}, 1}-\hat{p}_{\mathrm{e}, J}
$$

The net physical pressure drop follows as

$$
\Delta p=p_{\mathrm{f}}(0)-p_{\mathrm{e}}(L)=\rho U_{\mathrm{in}}^{2} \Delta \hat{p} .
$$

The other important performance metric is the uniformity of the porousmedia mass flux along the length of the channel pair. The local dimensionless flux is written as

$$
\hat{m}^{\prime \prime}=\Omega\left(\hat{p}_{\mathrm{f}}-\hat{p}_{\mathrm{e}}\right)
$$


The relative uniformity along the length of the channel pair can be represented as

$$
\Delta \hat{m}=\frac{\max \left[\hat{m}^{\prime \prime}\right]-\min \left[\hat{m}^{\prime \prime}\right]}{\max \left[\hat{m}^{\prime \prime}\right]} .
$$

Usually, the desired result is $\Delta \hat{m} \ll 1$.

Figure 3 is a composite contour plot that maps $\Delta \hat{p}$ and $\Delta \hat{m}$ as functions of $\Omega$ and $\Gamma$. The map shows that reducing the values of $\Omega$ and $\Gamma$ leads to good uniformity, but at the cost of higher pressure drop. The practical operating space is usually in the lower regions of the diagonal contours showing good flow uniformity. Most of the parameters that make up the dimensionless groups $\Omega$ and $\Gamma$ are physical parameters that are easily understood and evaluated. However, the parameter $K$ (Eq. 3) must be derived from the flow through the porous media. The generalization and evaluation of $K$ is discussed in the following sections.

Although the dimensionless map (Fig. 3) shows a great deal of summary information, it is also informative to consider a few profiles along the channel length. Figure 4a shows profiles of dimensionless velocities, pressures, and mass fluxes along the dimensionless channel length with $\Gamma=1000$ and $\Omega=10^{-2}$. As is always the case, the feed-channel velocity decreases monotonically and the exhaust-channel velocity increases monotonically. Under these conditions, the velocity profiles are found to be significantly nonlinear, with both upward and downward curvatures. The pressure drop is relatively large and also nonlinear. The mass flux between the channels varies greatly along the channel length, with a minimum near the mid-length of the channels. As seen from the black dot on the inset map (reproduction of Fig. 3), the flux nonuniformity is high and pressure drop is also high. Because the 
profiles and the characteristic parameters $(\Gamma$ and $\Omega$ ) are dimensionless, the profiles represent a wide range of profiles in physical (dimensional) terms.

The nonlinear behavior of the velocity and pressure profiles is caused by nonlinear physical interactions. The channel velocities are affected directly by mass exchange between the channels and the continuity equations (Eqs. 1 and 2). Pressure varies along the channel length owing to the momentum balance and viscous drag at the channel walls (Eqs. 8 and 9). The nonlinear interactions between velocity and pressure lead to possibly unanticipated results, such as mass-exchange minima along the channel length.

Figure $4 \mathrm{~b}$ shows profiles of dimensionless velocities, pressures, and mass fluxes along the dimensionless channel length with $\Gamma=1.0$ and $\Omega=0.5$. Assuming, for example, that the channel dimensions and inlet velocity are comparable to the results shown in Fig. 4a, the large increase in $\Omega$ is attributed to increased permeability of the porous media (i.e., $K$ ). Under these conditions, the shapes of the velocity profiles are qualitatively different from those in Fig. 4a (i.e., there are no inflections in the profiles). Because of the relatively low flow impedance through the porous media, the pressure variations are much smaller than those in Fig. 4a, and qualitatively quite different. Unlike the results shown in Fig. 4a, the pressure profile in the feed channel increases toward the exit end. The mass-flux profile increases monotonically, with a minimum near the inlet and maximum near the exit. Although the magnitude of the dimensionless flux in Fig. 4b is comparable to that shown in Fig. 4a, the behavior is qualitatively different.

Figure $4 \mathrm{c}$ shows profiles of dimensionless velocities, pressures, and mass fluxes along the dimensionless channel length with $\Gamma=1.0$ and $\Omega=10^{-2}$. 
Under these conditions, the flow impedance through the porous media is relatively large. This causes the pressures within the channels to be nearly spatially uniform. The result is nearly uniform mass flux between the channels, which is usually a desirable result. The velocity profiles are nearly linear, caused by the nearly uniform flux between the channels. For a particular $\Omega$, the magnitude of the mass flux can be increased by increasing $\Gamma$. However, if $\Gamma$ increases too greatly, the uniformity can be compromised (cf., Fig. 3). Another alternative for increasing mass fluxes, but retaining uniformity, is to reduce $\Omega$ and increase $\Gamma$. However, this comes at the cost of increasing overall pressure drop.

\section{Porous-media flow}

Flow through the porous media that connects the feed and exhaust channels is assumed to be represented as Darcy flow. In this case, the superficial velocity q may be represented as

$$
\mathbf{q}=-\frac{\mathcal{K}}{\mu} \nabla p
$$

where $\mathcal{K}$ is the porous-media permeability, $\mu$ is the fluid dynamic viscosity, and $p$ is the pressure. The conservation equation to be solved is

$$
\nabla \cdot \mathbf{q}=\nabla \cdot\left(-\frac{\mathcal{K}}{\mu} \nabla p\right)=0
$$

Assuming constant values of $\mathcal{K}$ and $\mu$, the Darcy problem reduces to

$$
\nabla^{2} p=0
$$

Solving this elliptic partial differential equation requires boundary conditions. 
Pressures are specified at the interface between the porous electrode and the floors of the flow channels. All other boundary conditions are specified as $\mathbf{n} \cdot \nabla p=0$, meaning that no flow crosses these boundaries. The interfaces with the bipolar plate and the membrane are essentially solid boundaries. The right and left boundaries are symmetry planes.

As with the channel-flow problem, the porous-media problem can be generalized in a dimensionless form. In this case, the two-dimensional Darcy problem uses the following dimensionless variables:

$$
\hat{p}=\frac{p}{p_{\mathrm{f}}-p_{\mathrm{e}}}, \quad \hat{x}=\frac{x}{W_{\mathrm{e}}}, \quad \hat{y}=\frac{y}{W_{\mathrm{e}}} .
$$

The pressure is normalized by the pressure difference between channels $\Delta p=$ $p_{\mathrm{f}}-p_{\mathrm{e}}$ and the spatial coordinates are normalized by the electrode width $W_{\mathrm{e}}$. Additionally, two geometric parameters are needed. The aspect ratio of the porous electrode can be represented as

$$
\gamma=\frac{H_{\mathrm{e}}}{W_{\mathrm{e}}}
$$

The ratio of channel width and electrode width can be represented as

$$
\delta=\frac{W_{\mathrm{c}}}{W_{\mathrm{e}}}
$$

With these definitions in mind, Fig. 5 illustrates the dimensionless electrode geometry.

In dimensionless variables, the conservation problem to be solved within the porous media is:

$$
\nabla^{2} \hat{p}=0
$$

The dimensionless pressure at the feed channel is $\hat{p}=1$ and at the exhaust channel is $\hat{p}=0$. All other boundaries must satisfy $\mathbf{n} \cdot \nabla \hat{p}=0$. It is important 
to note that the solution to the dimensionless Darcy-flow problem depends only on the dimensionless domain dimensions $\gamma$ and $\delta$.

Once the pressure field is determined for a particular geometry and porous media, the solution can be used to establish the parameter $K$ in Eq. 3. The process begins by evaluating the net flow rate $Q$ (per unit depth, $\mathrm{kg} \mathrm{m}^{-1}$ $\mathrm{s}^{-1}$ ) between the feed and exhaust channels, as

$$
Q=\rho \frac{\mathcal{K}}{\mu} \int_{0}^{H_{\mathrm{e}}} \frac{\partial p}{\partial x} d y,
$$

where $\rho$ is the fluid's mass density. Because the domain is symmetric, the integral can be evaluated at the mid plane of the porous electrode (i.e., at $\left.W_{\mathrm{e}} / 2\right)$. The mass flux $\left(\mathrm{kg} \mathrm{m}^{-2} \mathrm{~s}^{-1}\right)$, referenced to the full channel-floor width, follows as

$$
m^{\prime \prime}=\frac{2 Q}{W_{\mathrm{c}}} .
$$

Note that the flux from each feed channel supplies two exhaust channels, one on the left and one on the right (cf., Fig. 6). Thus, the "2" in the numerator accounts for the flow between adjacent channels. The parameter $K$ (Eq. 3) follows as

$$
K=\frac{m^{\prime \prime}}{p_{\mathrm{f}}-p_{\mathrm{e}}},
$$

where $p_{\mathrm{f}}-p_{\mathrm{e}}$ is the local pressure difference between the feed and exhaust channels. Because the problem is linear and the properties are assumed to be constant, the value of $K$ does not depend on the value of the pressure difference.

The parameter $K$ can now be written in terms of the solution to the dimensionless Darcy problem. The net mass flow rate at the electrode midplane 
290

291 292

(Eq. 38) becomes

$$
Q=\rho\left(\frac{\mathcal{K} \Delta p}{\mu}\right) \int_{0}^{\gamma} \frac{\partial \hat{p}}{\partial \hat{x}} d \hat{y}
$$

It then follows that

$$
K=\frac{2 \rho \mathcal{K}}{\mu W_{\mathrm{c}}} \int_{0}^{\gamma} \frac{\partial \hat{p}}{\partial \hat{x}} d \hat{y}=\frac{2 \rho \mathcal{K}}{\mu W_{\mathrm{c}}} \hat{Q}
$$

To simplify the nomenclature somewhat, it is convenient to define

$$
\hat{Q} \equiv \int_{0}^{\gamma} \frac{\partial \hat{p}}{\partial \hat{x}} d \hat{y}
$$

By solving the dimensionless Darcy problem for a wide range of $\gamma$ and $\delta$, a general expression for $\hat{Q}(\gamma, \delta)$ can be developed. The permeability $\mathcal{K}$ of a particular porous structure is needed to evaluate the parameter $K$. Permeability data is usually available from manufacturer's specifications, but can certainly be measured independently.

There are numerous approaches for solving the Darcy problem (Eq. 37), which is a linear, elliptic, partial differential equation in two-dimensional cartesian coordinates. The present study begins with a finite-volume discretization on a nonuniform $(201 \times 401)$ mesh network. The resulting algebraic equations are solved iteratively using a preconditioned conjugategradient method (incomplete LU preconditioner) as implemented in the MATLAB "bicgstab" function. Figure 7 shows representative solutions for fixed $\delta=0.4$ and three values of $\gamma$.

The Darcy problem is solved for 100 values of both $0.05 \leq \gamma \leq 1.5$ and $0.05 \leq \delta \leq 1$, producing a matrix of 10,000 total simulations. Figure 8 shows contour maps of $\hat{Q}(\gamma, \delta)$. For any combination of $\gamma$ and $\delta, \hat{Q}(\gamma, \delta)$ can be evaluated from the contour maps (the Appendix provides analytical fits to 
the contour maps). Then, the needed value of $K$ follows directly from Eq. 42 for any particular fluid and porous structure.

\section{Illustrative example}

To illustrate the use of the dimensionless analysis, consider a particular problem motivated by a redox flow battery. Table 1 lists a set of physical parameters as well as some of the derived dimensionless groups. Based on these parameters and operating conditions, the first step is to evaluate $\hat{Q}$ using Fig. 8 or the fits in the Appendix. In this case, $\hat{Q} \approx 0.2$, from which, using Eq. 42, the mass-flux parameter $K \approx 6 \times 10^{-4}$. Once $K$ is evaluated, the two dimensionless groups (Eqs. 16 and 17) describing the channel performance can be evaluated as $\Omega=1.5 \times 10^{-2}$ and $\Gamma=36.7$.

Using the values of $\Omega$ and $\Gamma$, Fig. 3 can be used to extract the dimensionless pressure drop through the channels and flow uniformity through the porous structure. Specifically, $\Delta \hat{p} \approx 10^{1.8}$ and $\Delta \hat{m} \approx 0.15$. For the parameters shown in Table 1, these circumstances lead to a net pressure drop of $\Delta p \approx 5300 \mathrm{~Pa}$, with approximately $15 \%$ variation in the local mass fluxes between channels.

Although $\Delta \hat{p}$ and $\Delta \hat{m}$ provide some very useful summary information, these results alone do not provide detailed information about profiles of velocity, pressure, and mass flux along the channels. Determining these details requires the full solution, which cannot be represented in a single graph. Nevertheless, the paper provides the information needed to program the solution. Assuming some working knowledge of computational mathematics and access to software such as MATLAB, writing the code is reasonably straightforward. 
Table 1: Nominal parameters for an illustrative problem

\begin{tabular}{ll}
\hline Fluid and flow & \\
Density, $\rho$ & $1350 \mathrm{~kg} \mathrm{~m}^{-3}$ \\
Viscosity, $\mu$ & $6 \times 10^{-3} \mathrm{~Pa} \mathrm{~s}$ \\
Inlet velocity, $U_{\mathrm{in}}$ & $0.25 \mathrm{~m} \mathrm{~s}^{-1}$ \\
\hline Channels & $1.5 \mathrm{~mm}$ \\
Width, $W_{\mathrm{c}}$ & $1.0 \mathrm{~mm}$ \\
Height, $H_{\mathrm{c}}$ & $100 \mathrm{~mm}$ \\
Length, $L_{\mathrm{c}}$ & $5 \mathrm{~mm}$ \\
Perimeter, $P_{\mathrm{c}}$ & $1.5 \times 10^{-6} \mathrm{~m}^{2}$ \\
Area, $A_{\mathrm{c}}$ & $1.2 \mathrm{~mm}$ \\
Hydraulic diameter, $D_{\mathrm{h}}$ & 1.5 \\
Aspect ratio, $\alpha$ & 14.8 \\
Ref & \\
\hline Porous electrode & $5 \mathrm{~mm}$ \\
Width, $W_{\mathrm{e}}$ & $1 \mathrm{~mm}^{-11} \mathrm{~m}^{2}$ \\
Height, $H_{\mathrm{e}}$ & 0.2 \\
Aspect ratio, $\gamma$ & 0.3 \\
Channel ratio, $\delta$ & \\
Permeability, $\mathcal{K}$ & \\
\hline & \\
\hline
\end{tabular}

\section{Dimensionless groups}

\begin{tabular}{ll}
$\Omega($ Eq. 16) & $1.5 \times 10^{-2}$ \\
$\Gamma($ Eq. 17) & 3.67 \\
\hline
\end{tabular}


A particular channel solution requires only a few seconds in a typical personal computer.

\section{Summary and conclusions}

This paper documents an analysis of the flow field within pairs of interdigitated channels that are connected by porous media beneath the channels. Although the physical model itself can be even more general, a particular objective here is to develop a model that can be expressed generally in dimensionless terms, with summary results being easily represented. Achieving this objective is facilitated by assuming incompressible and isothermal flow with constant properties. The solutions are represented in terms of three dimensionless groups, which involve fluid properties, channel geometry, porous-media configuration and properties, as well as operating conditions. Net pressure drop and flow non-uniformity are presented in terms of a single dimensionless contour map. However, the computational model itself is needed to evaluate more details about velocity, pressure, and mass flux profiles along the channel length.

\section{Acknowledgements}

This research is supported by the Office of Naval Research via grant N00014-12-1-0201. We gratefully acknowledge insightful discussions with Dr. Andrew Colclasure (ITN Energy Systems) on flow batteries and with Prof. Nils Tilton (CSM) on aspects of porous-media transport. 


\section{References}

[1] Q. Xu, T.S. Zhao, and P.K. Leung. Numerical investigations of flow field designs for vanadium redox flow batteries. Appl. Energy, 105:47$56,2013$.

[2] X. Ke, J.I.D. Alexander, J.M. Prahl, and R.F. Savinell. Flow distribution and maximum current density studies in redox flow batteries with a single passage of the serpentine flow channel. J. Power Sources, 270:646-657, 2014.

[3] C. Yin, Y. Gao, S. Guo, and H. Tang. A coupled three dimensional model of vanadium redox flow battery for flow field designs. Energy, 74:886-895, 2014.

[4] R.M. Darling and M.L. Perry. The influence of electrode and channel configurations on flow battery performance. J. Electrochem. Soc., 161:A1381-A1387, 2014.

[5] T. Jyothi Latha and S. Jayanti. Hydrodynamic analysis of flow fields of redox flow battery applications. J. Appl. Electrochem., 44:995-1006, 2014 .

[6] K.B. Shyam Prasad, S. Maharudrayya, and S. Jayanti. Flow maldistribution in interdigitated channels used in PEM fuel cells. J. Power Sources, 159:595-604, 2006.

[7] X.-D. Wang, Y.-Y. Duan, W.-M. Yan, and X.-F. Peng. Effects of flow channel geometry on cell performance for PEM fuel cells with parallel and interdigitated flow fields. Electrochimica Acta, 53:5334-5343, 2008. 
[8] P. Hu, L. Peng, W. Zhang, and X. Lai. Optimization design of slottedinterdigitated channel for stamped thin metal bipolar plate in proton exchange membrane fuel cell. J. Power Sources, 187:407-414, 2009.

[9] K.B. Shyam Prasad, P.V. Suresh, and S. Jayanti. A hydrodynamic network model for interdigitated flow fields. Intl. J. Hydrogen Energy, 34:8289-8301, 2009.

[10] X.-D. Wang, X.-X. Zhang, W.-M. Yan, D.-J. Lee, and A. Su. Determination of the optimal active area for proton exchange membrane fuel cells with parallel, interdigitated or serpentine designs. Intl. J. Hydrogen Energy, 34:3823-3832, 2009.

[11] G. Hu, J. Fan, S. Chen, Y. Liu, and K. Cen. Three-dimensional numerical analysis of proton exchange membrane fuel cells (PEMFCs) with conventional and interdigitated flow fields. J. Power Sources, 136:1-9, 2010.

[12] J. Lobato, P. Cañizares, M. A. Rodrigo, F.J. Pinar, and D. Úbeda. Study of flow channel geometry using current distribution measurement in a high temperature polymer electrolyte membrane fuel cell. J. Power Sources, 196:4209-4217, 2011.

[13] H.-W. Ku and H.-W. Wu. Influences of operational factors on proton exchange membrane fuel cell performance with modified interdigitated flow field design. J. Power Sources, 232:199-208, 2013.

[14] A.D. Santamaria, N.J. Cooper, M.K. Becton, and J.W. Park. Effect of channel length on interdigitated flow-field PEMFC performance: A com- 
putational and experimental study. Intl. J. Hydrogen Energy, 38:1625316263, 2013.

[15] A.D. Santamaria, J. Bachman, and J.W. Park. Design strategy for a polymer electrolyte membrane fuel cell flow-field capable of switching between parallel and interdigitated configurations. Intl. J. Hydrogen Energy, 38:5807-5812, 2013.

[16] H. Guo, M.H. Wang, J.X. Liu, Z.H. Nie, F. Ye, and C.F. Ma. Temperature distribution on anodic surface of membrane electrode assembly in proton exchange membrane fuel cell with interdigitated flow bed. $J$. Power Sources, 273:775-783, 2015.

[17] J.P. Feser, A.K. Prasad, and S.G. Advani. On the relative influence of convection in serpentine flow fields of PEM fuel cells. J. Power Sources, 161:404-412, 2006.

[18] Q. Ye, T.S. Zhao, and C. Xu. The role of under-rib convection in mass transport of methanol through the serpentine flow field and its neighboring porous layer in a DMFC. Electrochimica Acta, 51:5420-5429, 2006.

[19] C. Xu and T.S. Zhao. A new flow field design for polymer electrolytebased fuel cells. Electrochemistry Comm., 9:497-503, 2007.

[20] R.J. Kee, P. Korada, K. Walters, and M. Pavol. A generalized model of flow distribution in channel networks of planar fuel cells. J. Power Sources, 109:148-159, 2002. 
${ }_{423}^{421]}$ R.J. Kee, M.E. Coltrin, and P. Glarborg. Chemically Reacting Flow. $424 \quad$ Wiley, Hoboken, NJ, 2003. 


\section{Appendix A. Fits to summary results}

Figures 3 and 8 show summary results in graphical form. However, especially in a computational setting, it can be practically useful to have analytic representations of these results. The following sections report fits to the surfaces shown as contours in Figs. 3 and 8.

\section{Appendix A.1. Mass flow through porous structure}

The dimensionless mass flow rate $\hat{Q}(\gamma, \delta)$ can be fit using

$$
\hat{Q}(\gamma, \delta)=A_{\mathrm{Q}}\left[1-\exp \left(-\frac{\gamma}{B_{\mathrm{Q}}}\right)\right]
$$

where $A_{\mathrm{Q}}(\delta)$ and $B_{\mathrm{Q}}(\delta)$ are the 10th-order polynomials as

$$
A_{\mathrm{Q}}(\delta)=\sum_{i=0}^{10} a_{\mathrm{Q}, i} \delta^{i}, \quad B_{\mathrm{Q}}(\delta)=\sum_{i=0}^{10} b_{\mathrm{Q}, i} \delta^{i} .
$$

Table Appendix A.1 lists the polynomial coefficients $a_{\mathrm{Q}, i}$ and $b_{\mathrm{Q}, i}$.

\begin{tabular}{rrr} 
Table A.1: Fitting parameters for $\hat{Q}(\gamma, \delta)$ \\
\hline$i$ & $a_{\mathrm{Q}, i}$ & $b_{\mathrm{Q}, i}$ \\
\hline 0 & $1.101056 \times 10^{-01}$ & $1.152951 \times 10^{-01}$ \\
1 & $1.110693 \times 10^{+00}$ & $1.670473 \times 10^{+00}$ \\
2 & $1.492183 \times 10^{+01}$ & $-2.070772 \times 10^{+01}$ \\
3 & $-3.131708 \times 10^{+02}$ & $1.695987 \times 10^{+02}$ \\
4 & $2.461064 \times 10^{+03}$ & $-8.574868 \times 10^{+02}$ \\
5 & $-1.041577 \times 10^{+04}$ & $2.742538 \times 10^{+03}$ \\
6 & $2.624356 \times 10^{+04}$ & $-5.656151 \times 10^{+03}$ \\
7 & $-4.050821 \times 10^{+04}$ & $7.494034 \times 10^{+03}$ \\
8 & $3.757392 \times 10^{+04}$ & $-6.156397 \times 10^{+03}$ \\
9 & $-1.922792 \times 10^{+04}$ & $2.852407 \times 10^{+03}$ \\
10 & $4.172088 \times 10^{+03}$ & $-5.695567 \times 10^{+02}$ \\
\hline
\end{tabular}


444 445 ${ }_{447} \widetilde{\gamma} \leq 1$, and Table A.4 when $\widetilde{\gamma} \geq 1$. are fit using 10th-order polynomials as

Table A.2 lists the fitting coefficients.

\section{Appendix A.3. Mass-flux uniformity} 10th-order polynomials as,

446 The fitted polynomial coefficients $a_{\mathrm{m}, i j}$ and $b_{\mathrm{m}, j}$ are listed in Table A.3 for

\section{Appendix A.2. Net pressure drop}

The net pressure drop (i.e., between inlet to the feed channel and exit of the exhaust channel) can be fit using a 5th-order polynomial as

$$
\widetilde{p}(\widetilde{\omega}, \widetilde{\gamma})=\sum_{i=0}^{5} A_{\mathrm{p}, \widetilde{\omega}^{i}}
$$

where $\widetilde{p}=\log _{10}(\Delta \hat{p}), \widetilde{\omega}=\log _{10}(\Omega)$, and $\widetilde{\gamma}=\log _{10}(\Gamma)$. The coefficients $A_{\mathrm{p}, i}$

$$
A_{\mathrm{p}, i}(\widetilde{\gamma})=\sum_{j=0}^{10} a_{\mathrm{p}, i j} \widetilde{\gamma}^{j}
$$

Taking the logarithm of $\Delta \hat{p}, \Delta \hat{m}, \Omega$, and $\Gamma$, and denoting, $\widetilde{p}=\log _{10}(\Delta \hat{p})$, $\widetilde{m}=\log _{10}(\Delta \hat{m}), \widetilde{\omega}=\log _{10}(\Omega)$, and $\widetilde{\gamma}=\log _{10}(\Gamma)$, the function of uniformity $\widetilde{m}(\widetilde{\omega}, \widetilde{\gamma})$ can be fitted using the following expression,

$$
\widetilde{m}(\widetilde{\omega}, \widetilde{\gamma})=\left(\sum_{i=0}^{5} A_{\mathrm{m}, i} \widetilde{\omega}^{i}\right) \exp \left(-B_{\mathrm{m}} \widetilde{\omega}\right)
$$

where $A_{\mathrm{m}, i}$ and $B_{\mathrm{m}, i}$ are functions of $\widetilde{\gamma}$, and can be represented using the

$$
A_{\mathrm{m}, i}(\widetilde{\gamma})=\sum_{j=0}^{10} a_{\mathrm{m}, i j} \widetilde{\gamma}^{j}, \quad B_{\mathrm{m}}(\widetilde{\gamma})=\sum_{j=0}^{10} b_{\mathrm{m}, j} \widetilde{\gamma}^{j} .
$$
$\tilde{\gamma} \leq 1$, and Table A.4 when $\tilde{\gamma} \geq 1$. 


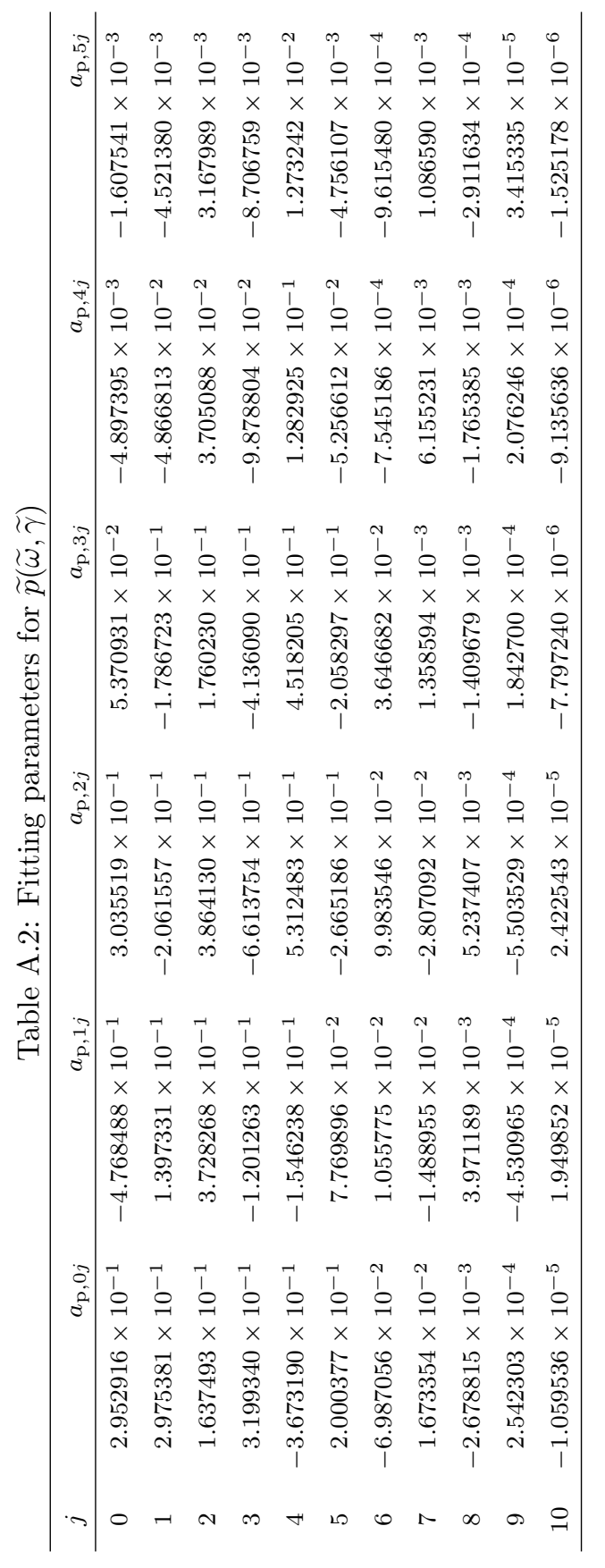




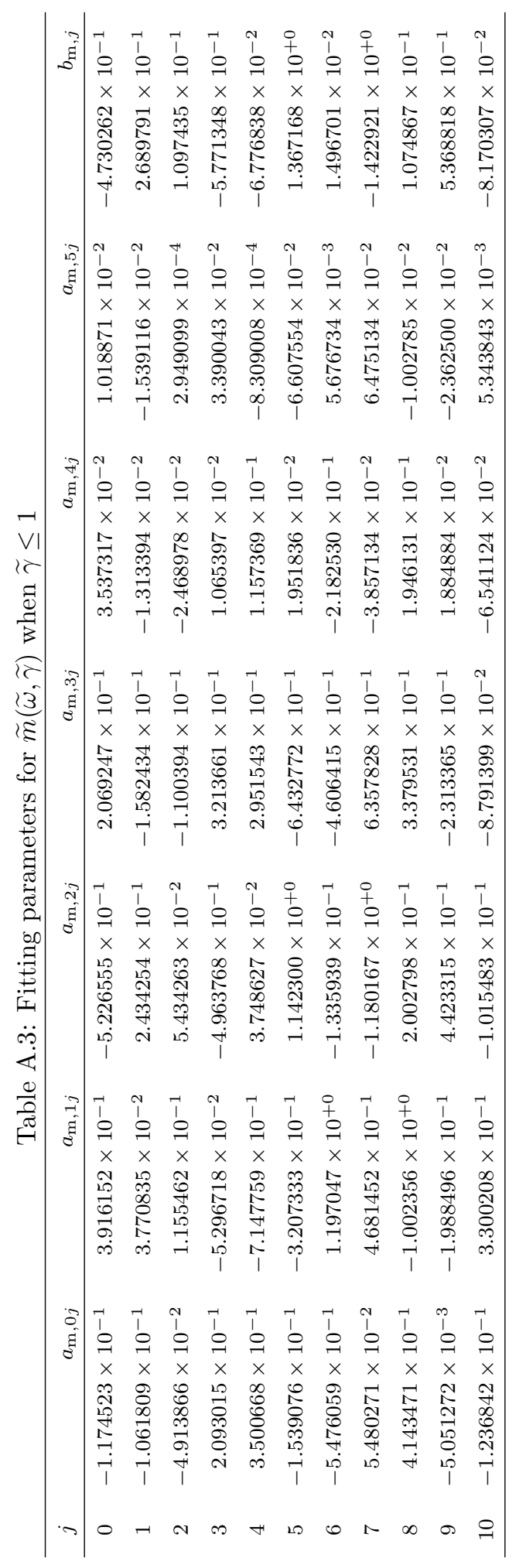




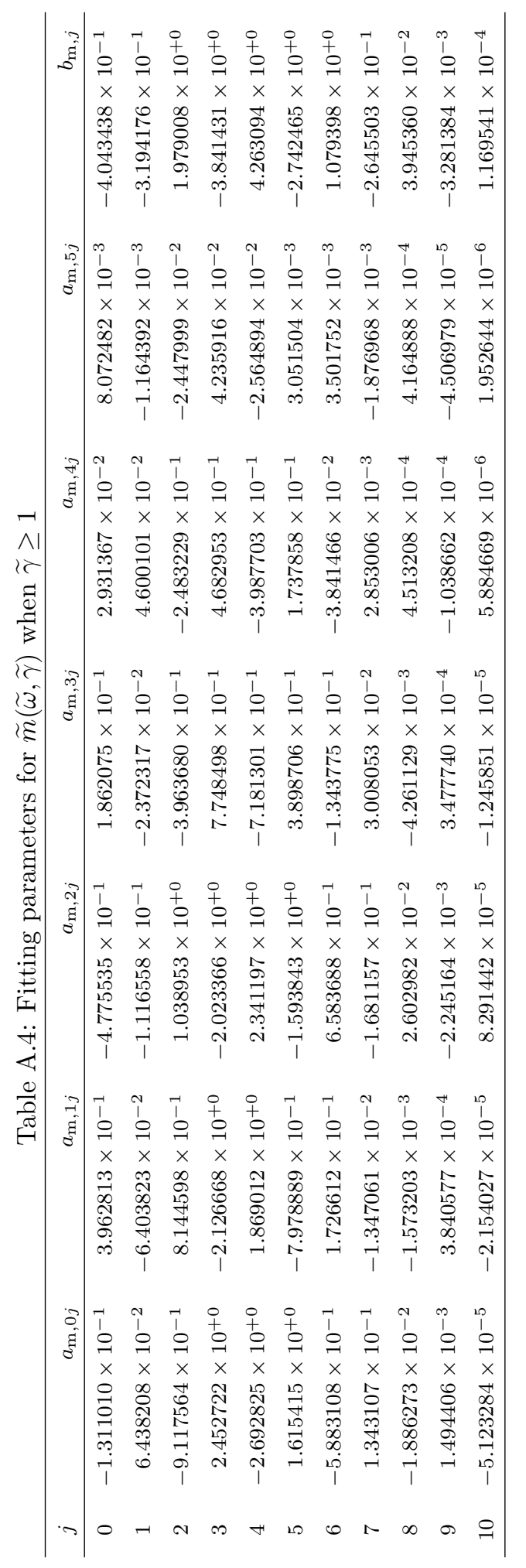




\section{List of Figures}

1. Illustration of a segment of redox flow battery with interdigitated channels in the bipolar plates. The anode and cathode structures are separated by ion-transport membranes.

2. Finite-volume stencil for modeling the two-channel interdigitated flow problem.

3. Dimensionless map of net pressure drop and porous-media flux uniformity.

4. Profiles of dimensionless velocities, pressures, and mass fluxes for three values of $\Gamma$ and $\Omega$. The black dots in the inset maps (cf., Fig. 3) show the position of the dimensionless channel and operating parameters.

5. Physical domain for modeling porous-media flow in dimensionless coordinates.

6. Illustration of the two-interdigitated-channel computational domain. The computational domain for the porous media is noted by the dashed line.

7. Example solutions of the dimensionless Darcy problem. The solutions illustrated here all use a channel width of $\delta=0.4$, but with three different values of the porous-media aspect ratio $\gamma$.

8. Contour maps of $\hat{Q}(\gamma, \delta)$ as functions of dimensionless porous media aspect ratio $\gamma$ and dimensionless channel width $\delta$. The upper panel shows the full $\gamma-\delta$ space. The lower panel shows an expanded region as marked by the dashed rectangle in the upper panel. 


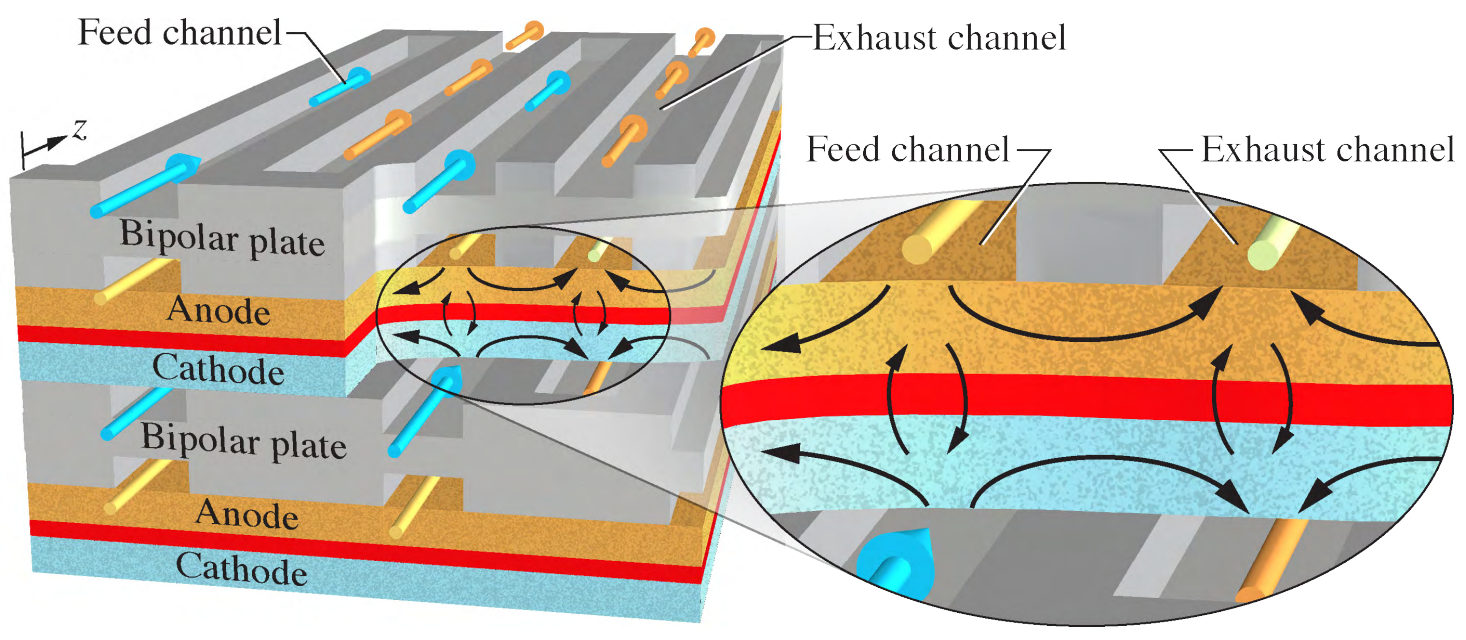

Figure 1: Illustration of a segment of redox flow battery with interdigitated channels in the bipolar plates. The anode and cathode structures are separated by ion-transport membranes. 
b) Top view
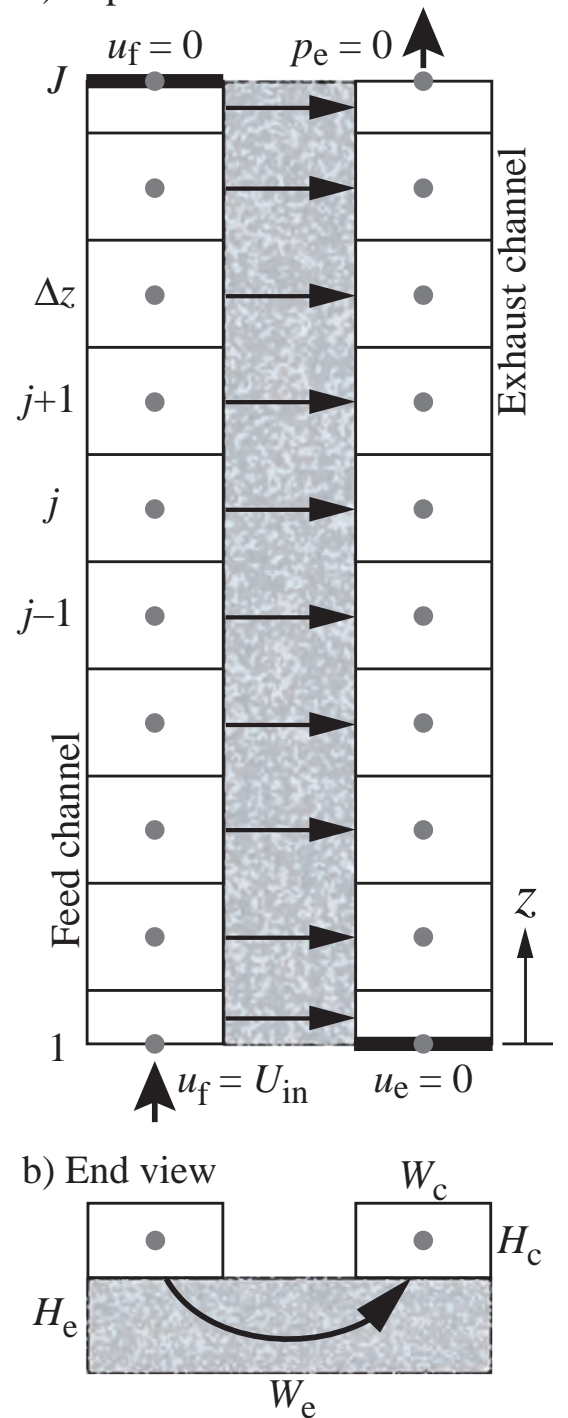

Figure 2: Finite volume stencil for the two-channel interdigitated flow problem. 


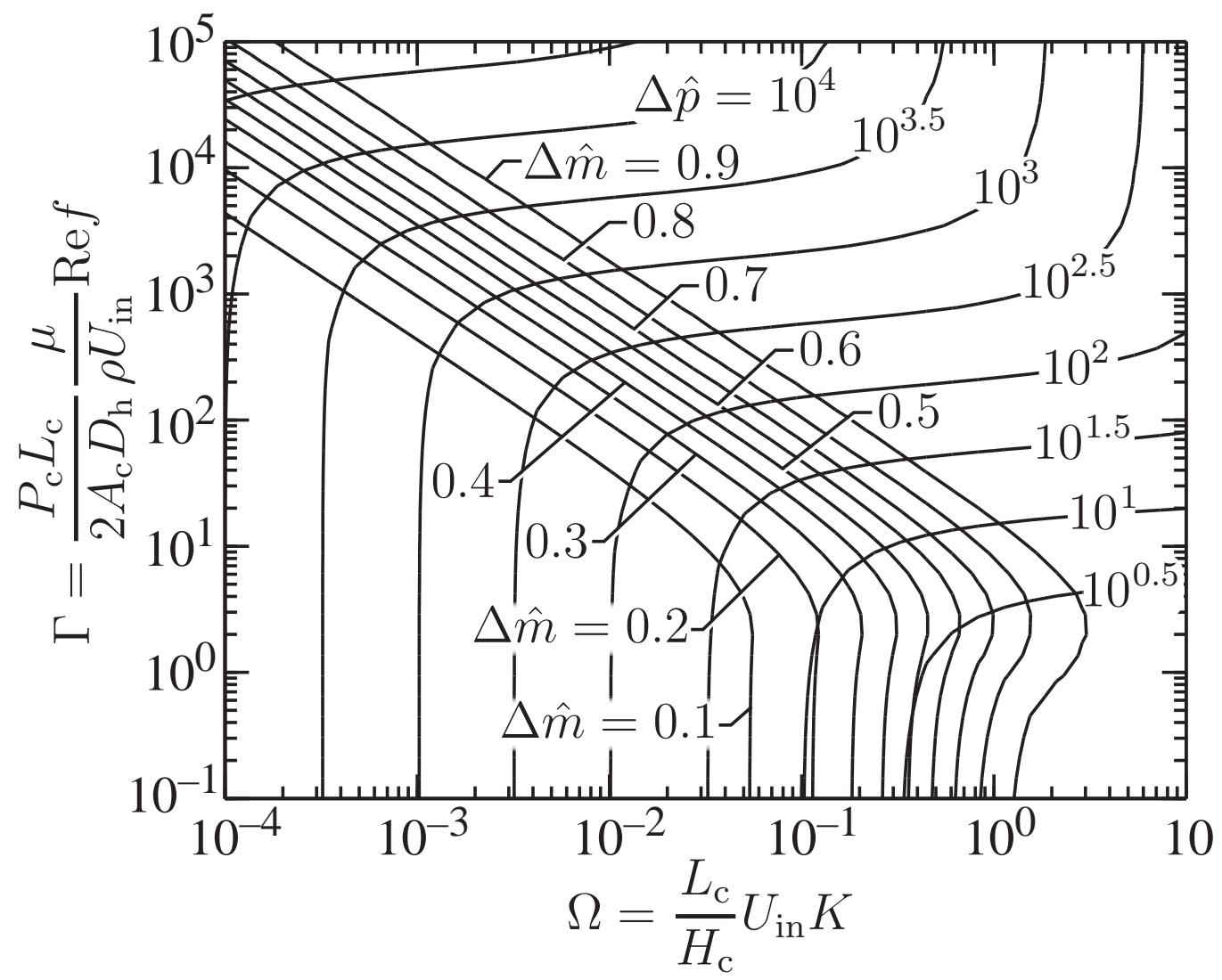

Figure 3: Dimensionless map of net pressure drop and porous-media flux uniformity. 

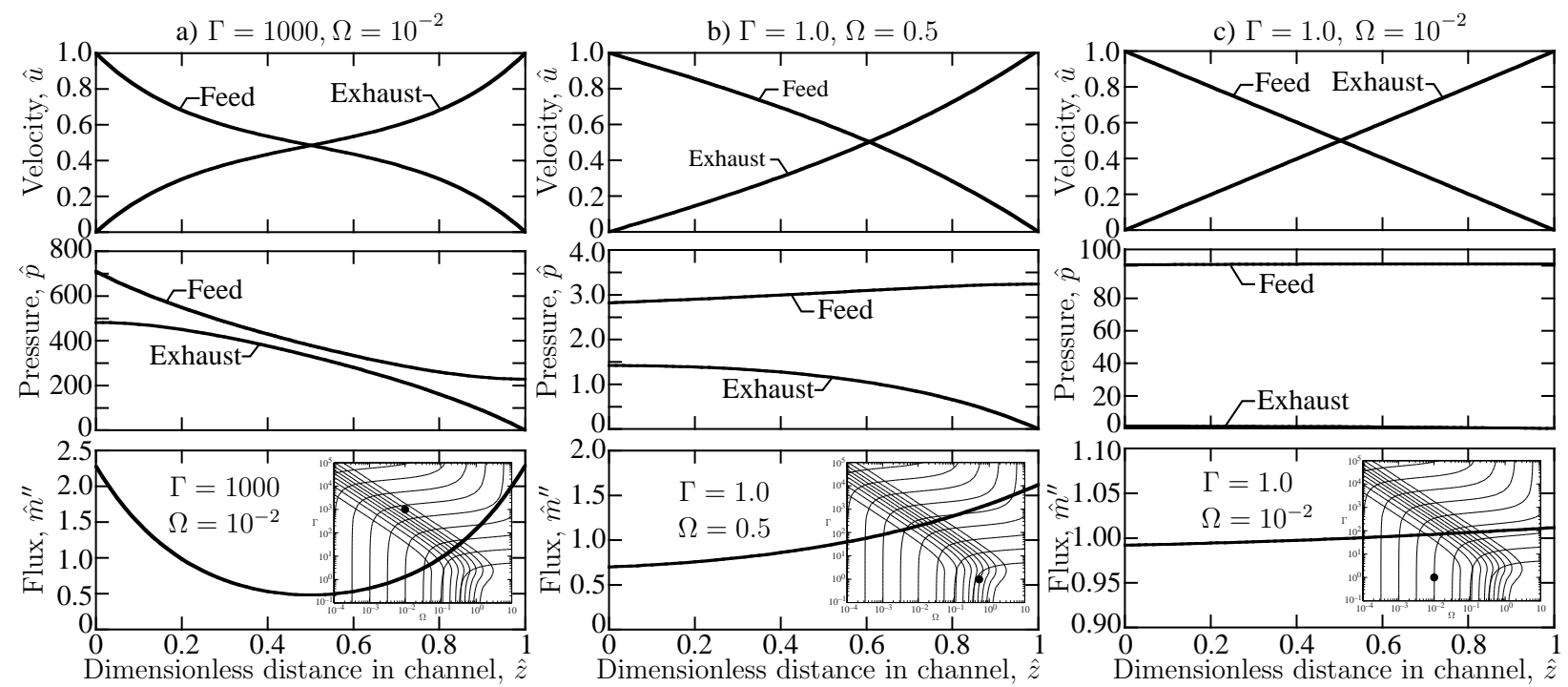

Figure 4: Profiles of dimensionless velocities, pressures, and mass fluxes for three values of $\Gamma$ and $\Omega$. The black dots in the inset maps (cf., Fig. 3) show the position of the dimensionless channel and operating parameters.

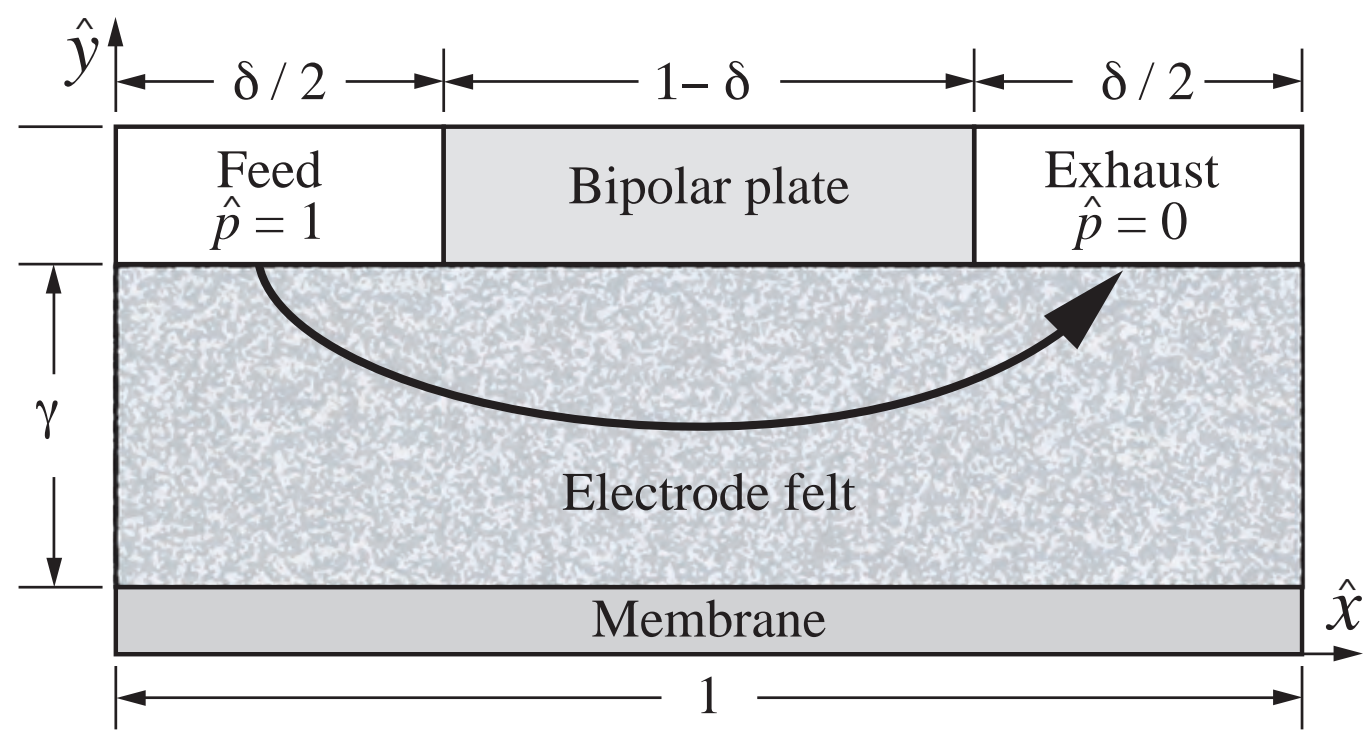

Figure 5: Physical domain for modeling porous-media flow in dimensionless coordinates. 


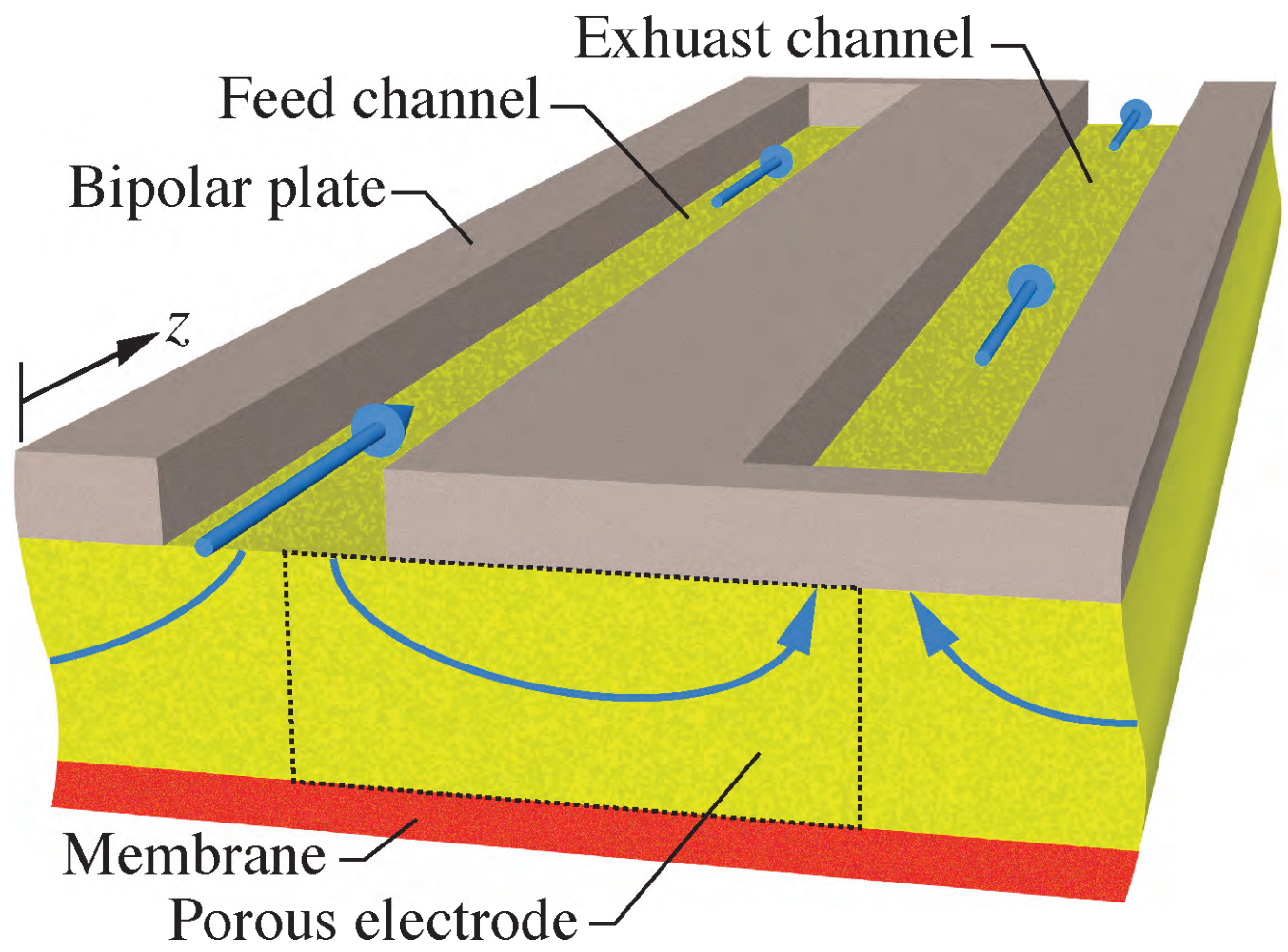

Figure 6: Illustration of the two-interdigitated-channel computational domain. The computation domain for the porous media is noted by the dashed line. 

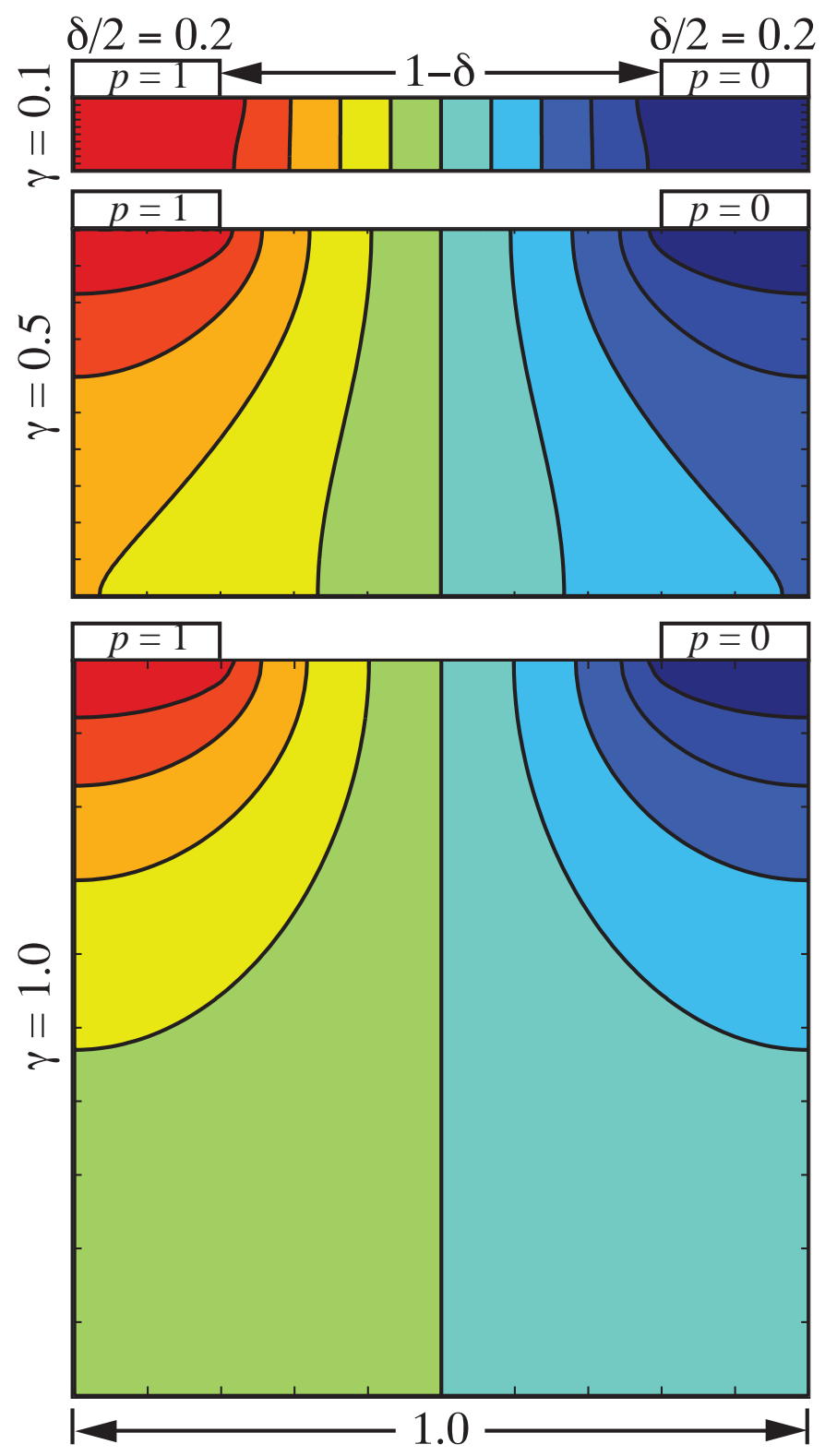

Figure 7: Example solutions of the dimensionless Darcy problem. The solutions illustrated here all use a channel width of $\delta=0.4$, but with three different values of the porous-media aspect ratio $\gamma$. 

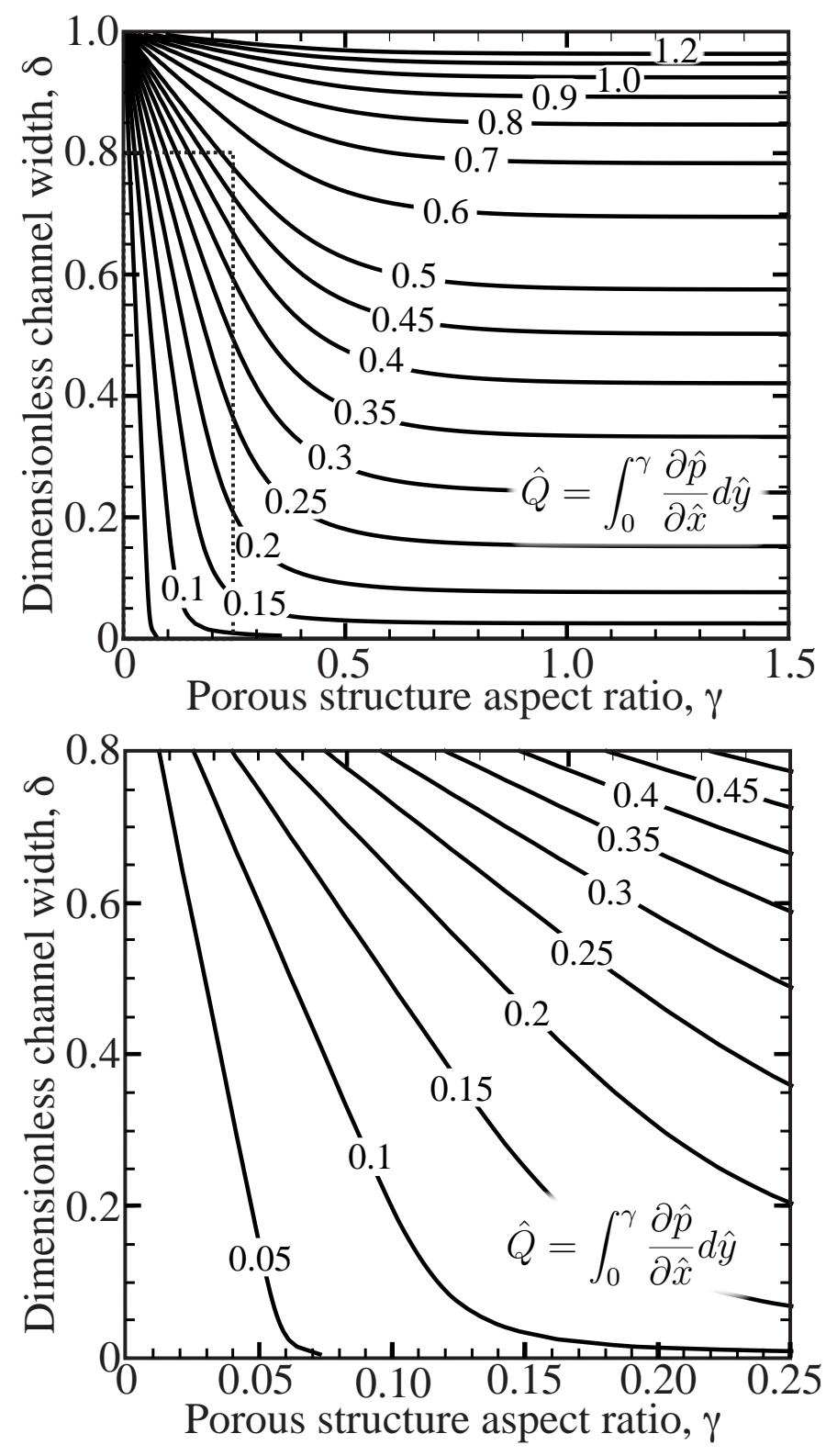

Figure 8: Contour maps of $\hat{Q}(\gamma, \delta)$ as functions of dimensionless porous media aspect ratio $\gamma$ and dimensionless channel width $\delta$. The upper panel shows the full $\gamma-\delta$ space. The lower panel shows an expanded region as marked by the dashed rectangle in the upper panel. 